\title{
SEVERE FALCIPARUM MALARIA - PATHOPHYSIOLOGY, CLINICAL MANIFESTATIONS/ COMPLICATIONS, AND MANAGEMENT CHALLENGES IN NIGERIA
}

\author{
NKPOZI MO* \\ Department of Medicine, Endocrinology, Diabetes and Metabolism Unit, Abia State University Teaching Hospital, Aba, Abia State, Nigeria. \\ Email: marcelnkpozi@gmail.com
}

Received: 12 October 2019, Revised and Accepted: 01 January 2020

\section{ABSTRACT}

Severe falciparum malaria remains a common cause of death among the under-5 Nigerian children despite Nigerians having partial immunity arising from their residence in a hyperendemic region for malaria transmission. It is, also, being reported in clinical practice among adolescent and adult Nigerians. The objective of this narrative review is to highlight the pathophysiology of severe malaria and relate that to its clinical manifestations/ complications and to highlight the challenges of severe malaria management in Nigeria. With a good understanding of the pathophysiology of severe malaria as it relates to its clinical manifestations, one expects a favorable outcome from the current treatment protocol.

Keywords: Severe falciparum malaria, Pathophysiology and clinical manifestations of severe malaria, Nigeria, Management challenges of severe malaria.

\section{INTRODUCTION}

Malaria in man is an infection caused by any of the following malarial parasites of the plasmodium species - Plasmodium falciparum, Plasmodium malariae, Plasmodium vivax, and Plasmodium ovale - which are spread to people through the bites of infected female Anopheles mosquitoes. P. falciparum is the most common of the parasites in most of the sub-Saharan Africa where it accounts for almost all the mortalities [1]. The global malaria burden is disproportionately borne by sub-Saharan Africa such that in 2015, the region had $90 \%$ of the global malaria cases and $92 \%$ of the malarial deaths [2].

Malaria can be severe or uncomplicated. According to the World Health Organization (WHO) [3], the criteria for severe malaria include that the malaria must be attributable to $P$. falciparum, the patient with the malaria is unable to swallow tablets, has evidence of, at least, one vital organ dysfunction or has a high malaria parasite count in his/her blood, and is at increased risk of dying. Additional WHO criteria [3] for the diagnosis of severe malaria from 2000 include impaired consciousness prostration or general body weakness, hyperparasitemia ( $>5 \%$ parasitized erythrocytes), hyperpyrexia (core body temperature $>40^{\circ} \mathrm{C}$ ), and hyperbilirubinemia (total bilirubin $>2.5 \mathrm{mg} / \mathrm{dl}$ ). Severe malaria is a potentially fatal but treatable disease.

As regard to the risk of severe malaria [3], it has been shown that in parts of the world where malaria is endemic, transmission of falciparum malaria is stable such that severe malaria is mainly a disease of children from the first few months of life to the age of 5 years. It is less common in older children and adults in those regions because they have partial immunity. In their study in Jos [4], Nigeria, 71\% of the children admitted for severe malaria were aged 5 years and below. Mortality in that study was associated with hypoglycemia, severe anemia, shock, and repeated prolonged seizures. Similarly, in their study in Enugu [5], Nigeria, 66.7\% of the children treated for severe malaria were under the age of 5 years with $8.8 \%$ of them being 6 months and below. In areas of the world with lower endemicity, severe malaria occurs in both children and adults. Travelers and migrant workers who do not have malaria immunity are at increased risk for severe malaria.

In some other studies, risk factors for severe malaria and death noted included age $>65$ years, female sex (especially when associated with pregnancy), non-immune status, coexisting medical conditions, no antimalarial prophylaxis, delay in treatment, and severity of the illness at admission (coma, acute kidney injury [AKI], shock, pulmonary edema, or coagulation disorders) [6-8]

Severe malaria is a major cause of death among children and often the major reason for children hospital admissions in sub-Saharan Africa. In Nigeria, malaria is one of the most important health problems [9], accounting for $25 \%$ of infant mortality, $30 \%$ of under -5 mortality, and $11 \%$ of maternal mortality. Severe malaria is a medical emergency that is rapidly associated with complications and death if prompt and appropriate treatment is not given.

\section{PATHOPHYSIOLOGY OF SEVERE MALARIA}

P. falciparum [10] attacks red blood cells (RBCs) at all stages of development unlike $P$. malaria which preferentially attacks old RBCs or $P$. ovale and P. vivax which prefer to attack reticulocytes (developing RBCs). In severe malaria, there is heavy parasitemia, in which the proportion of parasitized RBCs increases to more than $3 \%$ of the total RBCs.

The heavy load of $P$. falciparum in the peripheral blood [10] consumes and degrades the hemoglobin in the RBCs, the RBCs become irregular in shape, less deformable, adhere to venule and capillary endothelium to form cytoadherence, adhere to uninfected RBCs to form rosettes, and to other parasitized RBCs to form agglutination. Consumption and degradation of the RBCs hemoglobin, hemolysis of parasitized RBCs, blackwater fever, hypersplenism, and nutrients deficiency all lead to the severe anemia in complicated malaria. With massive hemolysis, lots of hemoglobin are liberated into the peripheral blood, are filtered in the renal glomeruli to cause hemoglobinuria.

The process of cytoadherence, rosetting, and agglutination is central to severe malaria pathogenesis [10]. Reduced deformability of the RBCs (parasitized) compromises their passage through the microcirculation resulting in reduced RBCs survival or lifespan, causes ischemic changes in the affected organs/tissues such that ensuing peripheral vasodilatation which occurs in an attempt to correct ischemia leads to systemic hypotension (shock). Shock in severe malaria could, also, be from sepsis which is a common associated feature of severe malaria.

With shock, anaerobic glycolysis occurs at the cellular level resulting to increased production of lactic acid, thus, leading to increased anion gap 
metabolic acidosis. Metabolic acidosis and hypoglycemia remain the main metabolic complications of severe malaria.

Exoerythrocytic phase of $P$. falciparum infection (in liver) causes decreased hepatic gluconeogenesis, leading to hypoglycemia Other causes of hypoglycemia in severe malaria include increased consumption of glucose by the falciparum parasites in RBCs and the increased pancreatic beta-cells stimulation by quinine and quinidine used in treating severe (complicated) malaria.

Microcirculation in the glomeruli of the kidneys is compromised by the processes of cytoadherence, rosetting, and agglutination resulting to pre-renal acute kidney failure which presents with oliguria and azotemia. Free hemoglobin filtered in the renal glomeruli, may, also, add to the AKI. Similarly, cerebral microcirculation is compromised, leading to ischemic changes and cerebral edema which manifest as confusion, convulsion, and coma seen in cerebral malaria.

Acute respiratory distress syndrome/acute pulmonary edema in severe malaria results from the hyperparasitemia itself, overhydration in the process of treating severe malaria, acute renal failure, and pregnancy. Hyperpyrexia in severe malaria is a consequence of cytokines and other pyrogens from lysed RBCs.

\section{CLINICAL FEATURES/COMPLICATIONS OF SEVERE MALARIA}

The major complications of severe malaria include cerebral malaria, acute renal failure, metabolic acidosis, hypoglycemia, acute respiratory distress syndrome/pulmonary edema, severe anemia, and/or bleeding. Any of these complications, once developed, can progress rapidly to death within hours to days and the complications can exist together or start within a few hours of the onset of another complication[3]. In no particular order, the complications in patients with severe malaria are discussed below.

\section{Cerebral malaria}

This is the most common clinical presentation of severe malaria as well as the most common cause of death in patients with severe malaria. In view of the aforementioned, some practitioners erroneously believe that cerebral malaria is synonymous with severe malaria. Cerebral malaria presents with confusion, drowsiness, and progressing to generalized convulsion and coma. Delirium, agitation, and even transient paranoid psychosis may develop as the patient recovers consciousness.

Cerebral malaria [11] is defined as the presence of $P$. falciparum parasitemia, in which the patient is unconscious with a Glasgow Coma Scale (GCS) score of 9 or less and other causes (e.g., hypoglycemia, bacterial meningitis, and viral encephalitis) are ruled out. Neck stiffness and focal neurologic deficits are absent and there is no specific EEG changes characteristic of severe malaria, but computed tomography and magnetic resonance imaging often show evidence of diffuse mild cerebral edema. Mortality of cerebral malaria ranges from $10 \%$ to $50 \%$ with treatment and most survivors have no neurologic deficits on discharge from hospitals [11].

\section{Pulmonary complications}

The first indication of impending pulmonary edema is breathlessness, followed by hypoxemia and Type 1 respiratory failure which may require intubation and mechanical ventilation. Pulmonary edema presents with $\mathrm{O}_{2}$ saturation $<92 \%$ on room air and a respiratory rate $>30 / \mathrm{min}$ and can be confirmed radiologically from a chest radiograph. Acute lung injury usually occurs few days after onset of severe malaria. Pulmonary edema in severe malaria is usually non-cardiogenic (no evidence of the left atrial hypertension) and may progress to acute respiratory distress syndrome with an increased pulmonary capillary permeability [12]. Volume overload in the course of intravenous (i.v) fluid administration can worsen pulmonary capillary leakage. Acute pulmonary edema rarely is seen with $P$. vivax and $P$. ovale malaria.

\section{Acute renal failure}

In severe malaria, AKI [13] usually presents with oliguria (urine volume $<400 \mathrm{ml}$ in $24 \mathrm{~h}$ ) or anuria (urine volume $<50 \mathrm{ml}$ in $24 \mathrm{~h}$ ) and azotemia (plasma creatinine is $>265 \mu \mathrm{mol} / \mathrm{l}[3 \mathrm{mg} / \mathrm{dl}]$ and blood urea $>20 \mathrm{mmol} / \mathrm{l}$ ). It is worth noting that hemoglobinuria resulting from massive intravascular hemolysis which manifests as the passage of dark red, brown, or black urine is transient and not a cause of AKI. This passage of dark-colored urine arising from massive intravascular hemolysis is called blackwater fever.

\section{Hypotension and shock}

This is usually associated with postural hypotension due to autonomic dysfunction. In the setting of severe malaria, cardiac pump function is usually preserved. Severe hypotension can be from pulmonary edema, metabolic acidosis, sepsis, and/or massive hemorrhage due to splenic rupture or bleeding into the gastrointestinal tract [13].

\section{Hypoglycemia}

This is a common feature of severe malaria which may be overlooked or missed because the clinical features of hypoglycemia (anxiety, breathlessness, tachycardia, sweating, coma generalized convulsions, etc.) are typical features of severe malaria per se. Hypoglycemia may be caused by quinine or quinidine which acts on the beta-cells of the pancreas to induce hyperinsulinemia [10].

\section{Severe malarial anemia}

Severe anemia is a common complication in the setting of severe malaria. In this regard, hemoglobin concentration is $<5 \mathrm{gm} / \mathrm{dl}$ or hematocrit is $<15 \%$ in children $<12$ years and in older children ( $>12$ years) and adults, a hemoglobin concentration of $<7 \mathrm{gm} / \mathrm{dl}$ or hematocrit $<20 \%$ defines severe anemia [14].

\section{Significant bleeding}

Some patients with severe malaria may present with recurrent or prolonged bleeding from the nose, gum, and venipuncture sites while some present with hematemesis or melena.

\section{MANAGEMENT OF SEVERE MALARIA}

Components of the management of severe malaria include clearance of the malarial parasites with specific antimalarial agents, diagnosis, and management of associated complications of severe malaria, additional treatment, and supportive care of the patient. The management of severe malaria begins with clinical assessment of the patient.

\section{Clinical assessments}

All patients with a diagnosis of severe malaria must be admitted in a hospital ward, preferably in an intensive care unit (ICU) setting if the facilities are available. If unconscious, the GCS score is determined at presentation and monitored regularly, an open airway is secured, breathing and circulation are assessed. Each patient being admitted for severe malaria is weighed or body weight is estimated for the purpose of antimalarial drug doses and fluids requirement. Thereafter, an i.v cannula is inserted for fluids administration and blood samples are collected for random blood glucose, serum electrolytes, urea and creatinine for renal function, hemoglobin concentration, blood film for malarial parasites, full blood count, venous lactate, arterial blood $\mathrm{pH}$ and gases, grouping and cross=matching a few pints of blood, clotting studies, platelet count, and blood culture for bacteria. Patient should, also, be assessed for fluid balance to avoid over- or underhydration. Cerebral malaria is not associated with signs of meningeal irritation such as neck stiffness, photophobia, or positive Kernig's sign.

It is important to note that in severe malaria, septicemia, pneumonia, and severe malaria may coexist [14]. There is a considerable clinical overlap between them such that in all cases of severe malaria, blood culture for bacteria is indicated. For the same reason, empirical parenteral broad-spectrum antibiotics should be started immediately with antimalarial treatment [14]. 
Specific antimalarial treatment

Parenteral antimalarial agents must be used in all cases of severe malaria. This should be followed by a full dose of effective artemisininbased combination therapy (ACT) orally. The two classes of drugs which are indicated in severe malaria are artemisinin derivatives (artesunate or artemether) and the Cinchona alkaloids [14] (quinine or quinidine).

Parenteral artesunate is the treatment of choice [14] and should be given to all adults and children (including infants, pregnant women in all trimesters, and lactating women) with a diagnosis of severe malaria through i.v or intramuscular routes for at least $24 \mathrm{~h}$. Once a patient has received, at least $24 \mathrm{~h}$ of parenteral artesunate and can tolerate oral therapy, treatment is completed with an ACT for 3 days.

Children weighing $<20 \mathrm{~kg}$ should receive $3 \mathrm{mg} / \mathrm{kg}$ body weight/dose of artesunate while bigger children (weighing $>20 \mathrm{~kg}$ ) and adults should receive $2.4 \mathrm{mg} / \mathrm{kg}$ body weight/dose of artesunate. Artesunate is dispensed as a powder of artesunic acid which is dissolved in 5\% sodium bicarbonate to form sodium artesunate. The latter is diluted in $5 \mathrm{ml} \mathrm{5 \%}$ dextrose and given i.v or intramuscular into the anterior thigh. Freshly prepared sodium artesunate is preferred and should not be stored for future use.

It is important to note that if artesunate is not available, intramuscular artemether is preferred to quinine for treating children and adults with severe malaria [14]. It is administered at a loading dose of $3.2 \mathrm{mg} / \mathrm{kg}$ body weight at presentation and $1.6 \mathrm{mg} / \mathrm{kg}$ body weight daily for the subsequent 5 days.

Quinine dihydrochloride is the most widely used of the quinine salts indicated in severe malaria. Quinine gluconate is less painful than quinine dihydrochloride when given by intramuscular injection while the sulfate is for oral administration. Quinine dihydrochloride is started with a loading dose of $20 \mathrm{mg}$ salt $/ \mathrm{kg}$ body weight with a maintenance dose of $10 \mathrm{mg} / \mathrm{kg}$ body weight given 8 hourly starting $8 \mathrm{~h}$ after the loading dose. If there is no improvement after $48 \mathrm{~h}$ on quinine, the dose is reduced to $10 \mathrm{mg} / \mathrm{kg}$ body weight every $12 \mathrm{~h}$. Each dose of quinine should be given as a slow rate controlled infusion (in 5\% dextrose water or saline) infused over $4 \mathrm{~h}$. If i.v infusion of quinine is not possible, it should be given by intramuscular injection to the anterior thigh; it should not be injected into the buttocks to avoid injury to the sciatic nerve. Note that, rapid i.v administration of quinine is dangerous and quinine is never given by i.v bolus injection as it causes fatal hypotension. Overdose of quinine is associated with deafness and blindness.

\section{Additional treatment and supportive care}

After a minimum of $24 \mathrm{~h}$ on parenteral antimalarial agent and once patient can tolerate oral drugs, the patient should be started on an effective ACT medication (such as artesunate+amodiaquine, dihydroartemisinin+piperaquine, or artemether+lumefantrine). ACTs containing mefloquine are avoided if patient presented with unconsciousness due to the risk of neuropsychiatric complications. Non-ACT-based combination regimen such as quinine+clindamycin or quinine+doxycycline can be used as additional treatment after parenteral antimalaria. Doxycycline is not given to children ( $<8$ years) and pregnant women.

Intensive nursing care as well as effective monitoring of vital signs, GCS score, blood glucose, and urine output are crucial in the management of severe malaria.

\section{Management of complications of severe malaria}

Diagnosis of the associated complications should be promptly made and treatment [14] commenced as follows at the same time that the parenteral antimalarial agents are given:

1. Severe malarial anemia - is treated by transfusion with screened fresh blood

2. Hypoglycemia - confirm by checking random blood glucose; correct and maintain normal blood glucose with dextrose infusion
3. Acute pulmonary edema - patient is nursed propped up at $45^{\circ}$ oxygen is given, loop diuretic is administered, and i.v fluids stopped. If need be (life-threatening hypoxemia), patient is intubated and positive pressure ventilation is given

4. Cerebral malaria (and unconsciousness) - maintain airway, lie patient on his/her side, exclude other causes of coma such as hypoglycemia and bacterial meningitis. Control convulsion with i.v or rectal diazepam, lorazepam, midazolam, or intramuscular paraldehyde

5. AKI - if patient has pre-renal AKI, fluid challenge may be helpful. Hemofiltration or hemodialysis is indicated in established acute renal failure

6. Shock - sepsis may be the cause; therefore, take blood for bacterial culture and sensitivity, give fluid to correct hemodynamic disturbances, and administer broad-spectrum antibiotics for sepsis

7. Spontaneous bleeding - transfuse with fresh whole blood

8. Metabolic acidosis - may require hemodialysis

9. Hyperpyrexia - use of antipyretic agent (e.g., parenteral paracetamol), ice-cold sponging, fanning, and use of cooling blanket.

\section{CHALLENGES IN THE MANAGEMENT OF SEVERE MALARIA IN NIGERIA}

Being in an endemic region for malarial transmission, severe malaria in Nigeria is seen mostly in children aged $<5$ years. This vulnerable age group is very dependent on the parent's health-seeking behavior to decide where and when to seek medical care when these children have a febrile illness. Some parents could equally decide not to seek any medical attention for their ill children. With the level of poverty in Nigeria, illiteracy, lack of effective health insurance scheme, and outof-pocket payment for medical services, patients with severe malaria present initially to medically unqualified health workers who often fail to make the correct diagnosis and undertreat the patients. By the time, the patients present to the qualified health-care professionals, they are moribund and in worse clinical states. Late presentation to the pediatrician at a time when the clinical features may have deteriorated so much creates difficulties with accurate diagnosis of severe malaria and its complications.

Second, ICU facilities in most tertiary hospitals in Nigeria are grossly inadequate to care for severe malaria and other critical medical, surgical, and obstetric conditions. These ICU facilities are practically not available in the primary and secondary health facilities in Nigeria where most children with severe malaria present initially.

Another major challenge to the successful management of severe malaria in Nigeria is the quality of antimalarial agents in the markets. A lot of low quality and substandard antimalarial agents imported by profit-motivated traders are in circulation and these are used unknowingly for treating cases of severe malaria. With this, the outcome of severe malaria treatment in Nigeria has remained poor.

Again, the workforce adequacy of Nigeria to deal with the problems of severe malaria is far from being enough. The pediatricians work mainly in the tertiary health facilities located in the cities while the health facilities in the hinterlands are manned by healthcare workers (doctors and nurses) who, most times, are not skilled and experienced enough to deal with severe malaria and its complications.

Finally, the laboratory support services needed to make accurate diagnosis of severe malaria and its complications are grossly lacking in the rural areas of Nigeria. Blood transfusion services are inadequate and the turnaround time for obtaining certain essential laboratory results (such as urea, creatinine, and lactate) is still more than $48 \mathrm{~h}$. Dialysis services are available, especially at the cities but are not affordable to all the patients.

\section{CONCLUSION}

Severe malaria is a potentially fatal medical emergency. In Nigeria, severe malaria still contributes significantly to childhood mortality. 
With a good grasp of the pathophysiology of severe malaria and how the complications arise, the health-care professionals should be in a position to offer treatment that will be associated with favorable outcome. The challenges to the successful management of severe malaria in Nigeria are enormous but with the right government policy, good health funding, and capacity building, morbidity and mortality from severe malaria in Nigeria can be considerably reduced.

\section{REFERENCES}

1. Snow RW, Omumbo JA. Malaria. In: Jamison DT, Feachem RG Makgoba MW, Bos ER, Baingana FK, Hofman KJ, editors. Disease and Mortality in Sub-Saharan Africa. $2^{\text {nd }}$ ed., Ch. 14. Washington, DC: The International Bank for Reconstruction and Development/ The World Bank; 2006.

2. World Health Organization. World Malarial Report 2018. Geneva: World Health Organization; 2018.

3. World Health Organization. Severe falciparum malaria, World Health Organization, Communicable Diseases Cluster. Trans R Soc Trop Med Hyg 2000;94 Suppl 1:S1-90.

4. Angyo IA, Pam SD, Sziachetka R. Clinical pattern and outcome in children with acute severe falciparum malaria at Jos University Teaching hospital, Nigeria. East Afr Med J 1996;73:823-6.

5. Edelu BO, Ndu IK, Igbokwe OO, Iloh ON. Severe falciparum Malaria in children in Enugu, South East, Nigeria. Niger J Clin Pract 2018;21:1349-55

6. Bruneel F, Hocqueloux L, Alberti C, Wolff M, Chevret S, Bedos JP, et al. The Clinical spectrum of of severe imported falciparum malaria in the ICU: Report of 188 cases in adults. Am J Resp Crit Care Med 2003;167:684-9.

7. Blumberg L, Lee RP, Lipman J, Beards S. Predictors of mortality in severe malaria: A two year experience in a non-endemic area. Anaesth Intensive Care 1996;24:217-23.

8. Schwartz E, Sadetzki S, Murad H, Raveh D. Age as a risk factor for severe Plasmodium falciparum malaria in non-immune patients. Clin Infect Dis 2001;33:1774-7.

9. National Population Commission, National malaria Control Programme, ICF International. Nigeria Malaria Indicator Survey 2010 Abuja, Nigeria: NPC, NMCP, and ICF International; 2012.

10. White NJ, Breman JG. Malaria and babesiosis. In: Kasper DL, Braunwald E, Fauci AS, Hauser SL, Longo DL, Jameson JL, editors. Harrison's Principles of Internal Medicine. 18 ${ }^{\text {th }}$ ed., Vol. 323. New York: McGraw Hill; 2011. p. 1218-32.

11. Warrell DA, Looareesuwan S, Warrell MJ, Kasensarn P, Intaraprasert R, Dunnag D, et al. Dexamethasone proves deleterious in cerebral malaria. A double-blind trial in 100 comatose patients. N Engl J Med 1982;306:313-9.

12. Gachot B, Wolff M, Nissack G, Veber G, Vachon F. Acute lung injury complicating imported Plasmodium falciparum malaria. Chest 1995;108:746-9.

13. Trampuz A, Jereb M, Muzlovic I, Prabhu RM. Clinical review severe malaria. Crit Care 2003; 7:315-23.

14. World Health Organization Guidelines for the Treatment of Malaria. $3^{\text {rd }}$ ed., Ch. 7. Geneva: World Health Organization; 2015. 